\title{
WIRELESS INFANT MONITORING SYSTEM BASED ON VIDEO OBJECT TRACKING AND ENVIRONMENTAL SENSORS
}

\author{
Ms. Maitrayee Mahanta \\ MCA scholar,School of CS \& IT, Dept of MCA, Jain (Deemed-to-be) University, \\ Bangalore
}

Article DOI: https://doi.org/10.36713/epra4326

\begin{abstract}
Many research has been going on for the development of better infant monitoring system to detect the cause of SIDs (Sudden Infant Deaths) among children and also to monitor or prevent infants from facing mechanical accidents or environmental discomfort. The proposed infant monitoring system is designed to detect the motion and the temperature of the infant using various IOT devices. A camera module is also used to capture videos which allows family members to monitor infant through videos. The data collected from all the sensors will be analyzed and hence will send an alert notification to the parents or relatives of the infant if found any abnormality in the measurements of the taken by the sensors. The technique also focuses on making the whole process of monitoring the infant portable. Which means users can easily monitor their kids from anywhere they want to without having their physical presence .

KEYWORDS-IoT, Infant Monitoring System, Sensors, Cloud Computing
\end{abstract}

\section{INTRODUCTION}

According to a recent survey, almost 30 percent of infants die in a year due to heat stroke or some other mechanical and environmental accidents every year around the world.

Most of the time the reason behind an infant's death is unknown, and hence the syndrome is termed to as Sudden Infant Death Syndrome. Infants mostly die during their sleep without showing any pre-symptoms.

Also they might face mechanical accidents by moving into places which we consider dangerous for them in the absence of parents or other family members. As infants between 0-3 years cannot speak on their own even, we can't analyze it before if the kid is facing any discomfort .

There are also various evidences available for death or illness of small children because of heat stroke caused while they were inside vehicles. Parents or others might not realize the intensity of the temperature, whether it is harming their baby or not and they might continue to be in the vehicle with them . Parents might also leave their children inside their vehicle for some reason which can also be proved to be hazardous for kids as it doesn't takes much time for a vehicle to get heat up.

Body of small kids heats up much faster than the adults and hence have greater susceptibility of getting harmed. According to National Highway Traffic Association in US, around 661 children have already died between 1998-2015 because of heatstroke in cars.

Heatstroke is truly a very devastating disease that happens suddenly, resulting in several disabilities and deaths, hence needs to be detected and prevented from occurring.

As mentioned earlier, mechanical accidents may also occur if a child is left alone inside a car due to any unexpected conditi

To overcome all the issues, the above mentioned technique has been proposed which makes use of various technologies such as IoT and cloud computing. As we know, with the introduction of the Internet of Things (IoT), invention and development of devices such as this has become easier and more efficient. The IoT has enabled various mechanical and digital devices to work more efficiently. The devices are now able to integrate and communicate with each other via Internet. 


\section{SJIF Impact Factor: 6.260| ISI I.F.Value:1.241| Journal DOI: 10.36713/epra2016 ISSN: 2455-7838(Online) EPRA International Journal of Research and Development (IJRD) - Peer Reviewed Journal}

There are various levels of IoT, the most basic implementation of the IoT devices where they are connected just to collect sensor data is considered to be level 1. In the proposed model we are going to use level 4 of IoT which includes the integration of special technology such as cloud computing which allows the use of IoT devices up to a next level.

With cloud computing, people are able to access their resources from anywhere in the world, at any time as cloud providers are meant to provide services $24 * 7$ to its users, with the help of users. Easy accessibility and security of data in cloud has made it the most emerging technology in recent times.

In the proposed technique, two sensors are used temperature sensor and motion sensor. The temperature sensor will monitor the temperature of the infant in Celsius anytime, even when parents are not near them and will send notification to parents if the temperature goes above what is considered to be abnormal.

Similarly, the motion sensor will detect any motion of the baby that happens around it . It will detect the time when the motion happened and also when it ended. This sensor, hence can be installed in places that is considered dangerous.

Both the sensors can be connected to special microcontrollers which are available in the

market such as Arduino, Raspberry pie etc which allows us to program the sensors and make them work or behave as we want using different codes and algorithms.

The proposed system can also be installed inside cars where it will constantly monitor the temperature and motion of the infant and will keep the parents alert all the time, thus minimizing the cause of heat stroke or other accidents in infants due to temperature difference inside the car or some other unlikely incident .

A camera module is also used to monitor the infants by watching videos captured through it.

The parents or any other family member of the infant can access and analyze the data collected through all the modules used above by logging in to an application created for the particular system.

An Wi-Fi module is also used to connect all the devices to the internet so that the data collected through them can be pushed into cloud in a database where it can analyzed and accordingly can accessed by the users .

The entire system can be integrated with cloud using various tools, APIs and the internet .

\section{OBJECTIVES}

1. To develop a better infant monitoring system using various IOT devices

2. To develop a portable monitoring tool for parents to monitor their infant from any place using cloud technology

3. To develop a secure interface to the monitoring system so that only the authorized and deserved person have access to all the data to monitor the infant by using an authentication mechanism .

4. To provide an efficient monitoring system for parents so that they get every notifications in detail

\section{PROBLEMS WITH EXISTING SYSTEMS}

1. Manual or traditional monitoring systems takes more time to realize the problems faced by the infant

2. Systems are not portable.

3. Data collected from the sensors are not analyzed

\section{LITERATURE REVIEW}

${ }^{[1]}$ In this paper the author quoted that the current research on the development of infant monitoring system focuses on portability and compactness.

[2] This paper suggests ways to develop wearable monitoring systems for infant's which can be integrated with the baby's clothes or shoes and hence the system can be used at home to keep track of baby's health without going to hospitals every time

[3] In this paper the author proposes a model which uses parameters like infant's heart beat and body position to build a system to detect and prevent SIDs among infant's

${ }^{[4]}$ In this paper the author proposes a model based on Arduino which uses GSM module to build an efficient infant monitoring systems

\section{PROBLEM STATEMENT}

Many children die unknowingly due to unpredictable heat stroke, illness or accidents because of mechanical and environmental factors.

Without proper detection and alert system, it is difficult to be aware of the problems facing by the children, specially between the age of 0-3 years, as they cannot convey anything to anyone even when they are facing any discomfort. As majority of parents, also nowadays, have started leaving their kids alone because of some important reasons such as jobs etc, they cannot be with the kid all the time . during their absence, the care takers or other person around them might not also be conscious enough to detect difficulties faced by the children at the earliest.

\section{SOLUTION}

The proposed system aims to overcome the limitation present in the existing system. In this model, the parents or the users of the system is logged in to an application first using a secure authentication mechanism. After getting access to the application the users can monitor the movement as well as health of the infant wirelessly from anywhere .

Various sensors such as motion and temperature sensors are used to monitor the baby from all aspects

The system is also designed to be a low cost tool so that every parents can use it to monitor their baby. Because of cloud integration, the system offers greater mobility, allowing users to access the system using mobile applications from anywhere . 


\section{EPRA International Journal of Research and Development (IJRD)}

Volume: 5 | Issue: 4 | April 2020

- Peer Reviewed Journal

Hence, the proposed system can prove to be more

portable and efficient, where user can receive alerts based on analyzed data collected from various sensors used in the model and stored in cloud. Live video capturing of the infant using camera module will help user to get real time video monitoring of the infant.

Users can also access previous alert notifications and the data collected by sensors later as the data will always be stored and protected in cloud via internet.

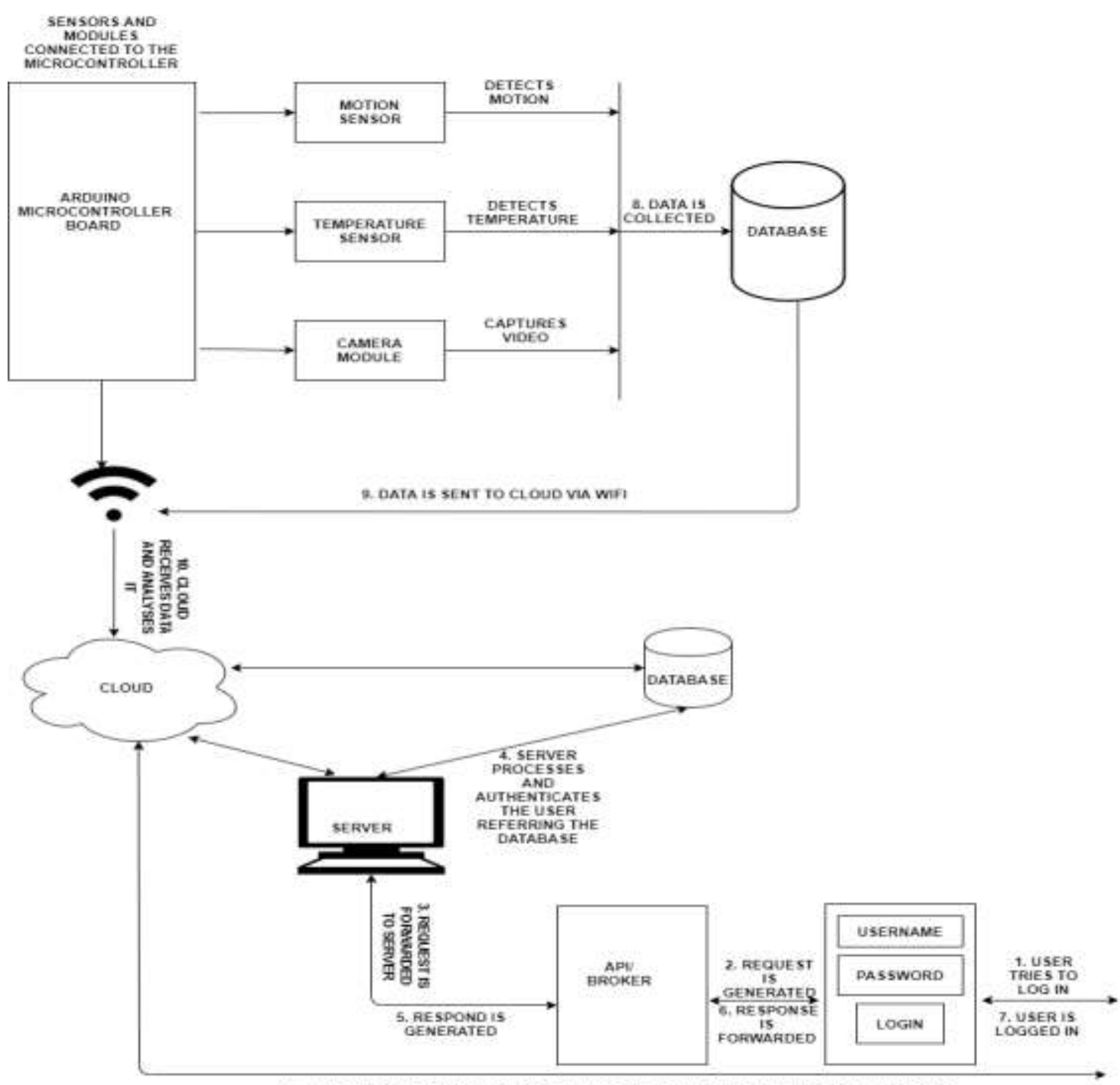

11. ALERT IS GENERATED AND SENT AS NOTIFICATION TO USER VIA THE USER INTERFACE

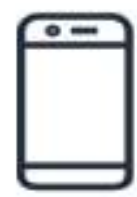

Mobile client

FLOW DIAGRAM OF WIRELESS INFANT MONITORING SYSTEM BASED ON VIDEO OBJECT TRACKING AND ENVIRONMENTAL SENSORS

\section{Figure 1 : System Architecture}

\subsection{WORKING}

- Parents can log into an application designed specifically to receive alerts from the system using their credentials.

- Secure authentication mechanism is used to ensure that only the authorized person should get all the details of the baby.

- Whenever the baby is around, the temperature and motion is detected. The data is collected and pushed into a database in cloud

- The Camera module will also capture video and will upload it in cloud database.

- Data collected from the sensors is analyzed and stored in cloud using various data analysis algorithms.

- When any abnormality is detected, an alert notification is sent to the authorized users 
through the application used as an user interface.

- All the sensors used in the system can be programmed using Arduino board, to which all the sensors will be connected,

- The Wi-Fi module will help connect the device with internet, making the whole system portable and wireless.

\section{VII.FUTURE ENHANCEMENTS}

Sensors can be configured to capture and analyze infants steps, so as to determine their movement more efficiently using various machine learning techniques.

Various other sensors like pulse detection sensor, vibration sensor etc can be used to increase the number of metrics based on which a baby can be monitored.

\section{CONCLUSION}

- Use of an wireless infant monitoring system can enable saving of majority of infants from sudden deaths or illness .

- It can also help parents or medical experts to determine the causes of any abnormality of children by analyzing and studying the real time data captured by the system.

- The easy portability of the system can allow parents to be more cautious even when they are not near their baby.

- Use of application interface and authentication mechanism can also prevent unauthorized person from gaining access to the data.

\section{REFERENCES}

1. Author: Budiman P.A. Rohman, Muhammad Bagus Andra, Masahiko Nishimoto Towards a Compact Infant Monitoring System using UWB Radar and Environmental Sensors

2. Author: Yves Rimet, Yves Brusquet, Dominique Ronayette, Christian Dageville, Marc Lubrano, Eric Mallet, Caroline Rambaud, Christian Terlaud, Jerome Silve, Olivier Lerda Larissa I. Netchiporouk, JeanLuc WeberSurveillance of infants at risk of apparent life threatening events (ALTE) with the BBA bootee: a wearable multiparameter monitor

3. Author : E.Abirami and S. Karthika Design of Infant Monitoring System to reduce Risk of Sudden Infant Death Syndrome

4. Author : Abdel Rahman AlkharabshehAn Intelligent Mobile Agents System for Sudden Infant Death Syndrome Monitoring 